\title{
Imbalance of M1/M2 macrophages is linked to severity level of knee osteoarthritis
}

\author{
BAOLONG LIU ${ }^{1}$, MAOQUAN ZHANG ${ }^{2}$, JINGMING ZHAO $^{3}$, MEI ZHENG $^{4}$ and HAO YANG ${ }^{1}$ \\ Departments of ${ }^{1}$ Emergency Surgery, ${ }^{2}$ Rheumatology and ${ }^{3}$ Spine Surgery, The Affiliated Qingdao Hiser Hospital \\ of Qingdao University (Qingdao Hospital of Traditional Chinese Medicine), Qingdao, Shandong 266001; \\ ${ }^{4}$ Department of Internal Medicine, People's Hospital of Zhangqiu, Jinan, Shandong 250200, P.R. China
}

Received March 19, 2018; Accepted September 11, 2018

DOI: $10.3892 /$ etm.2018.6852

\begin{abstract}
Macrophages, whether M1 or M2 subtype, have been found to be implicated in the pathogenesis of osteoarthritis (OA). However, no study regarding the status of M1 and M2 macrophages has been reported in knee OA. To investigate the status of M1 and M2 macrophages in knee OA, synovial fluid as well as peripheral blood were collected from 80 patients with knee OA and 80 healthy controls. Reverse transcriptionquantitative PCR was used to quantitatively detect the expression of CD11c as a marker for M1 macrophages and CD206 as a marker for M2 macrophages from synovial fluids. As confirmation, flow cytometry was employed to count the number of monocytes from whole blood using the CD86 (M1) and CD163 (M2) markers on monocytes. The ratio of M1 to M2 macrophages was shown to be markedly higher in knee OA than that of control and that the ratio was significantly positively correlated with level of Kellgren-Lawrence grade in knee OA, that is, the higher the ratio the more severe the knee OA seems to be. Thus, our study presented direct evidence for the involvement of macrophages in the pathogenesis of knee OA.
\end{abstract}

\section{Introduction}

Osteoarthritis (OA) was histologically thought of as regular 'wear and tear' disease whose immune system was unlikely to be affected (1). With the understanding of pathogenesis of $\mathrm{OA}$, it is now generally accepted to be a low-grade inflammatory disease affecting the whole joint (2). Although the pathogenesis and progression of OA seem to be the result of the complex interplay between mechanical, cellular and inflammatory factors, the mechanism remains unknown (3).

Correspondence to: Dr Hao Yang, Department of Emergency Surgery, The Affiliated Qingdao Hiser Hospital of Qingdao University (Qingdao Hospital of Traditional Chinese Medicine), 4 Renmin Road, Qingdao, Shandong 266001, P.R. China

E-mail: hwq2tf@163.com

Key words: macrophage, osteoarthritis, knee, synovial fluid
Knee OA is the most common type of OA, and also the most common cause of disability of people worldwide. Deterioration of articular cartilage is clinically regarded as the main cause leading to knee OA (4), but the impairment of chondrogenesis from inflammation remains elusive and deserves to be elucidated. Despite this, macrophage polarization $(5,6)$ has been documented to be implicated in the deterioration of cartilage. Especially, M1 macrophage has been identified as the major mediator in the anti-chondrogenic process (7). In consideration of the two main subtypes of macrophages (8), M1 macrophage is believed to be pro-inflammatory whereas M2 macrophage anti-inflammatory, together with a previous relevant report (6); we hypothesized that the balance between M1 and M2 macrophages might be skewed in knee OA. To test our hypothesis, we measured the ratio of M1 to M2 macrophages from synovial fluids and peripheral blood from patients with knee OA and matched healthy controls. It was found that imbalance of M1/M2 macrophages occurs in knee OA and the degree of imbalance was associated with severe level of knee $\mathrm{OA}$, suggesting that re-balance of the ratio might be used as a novel therapeutic alternative for knee OA.

\section{Materials and methods}

Clinical samples. This study was approved by the Medical Ethics Committee of The Affiliated Qingdao Hiser Hospital of Qingdao University (Qingdao Hospital of Traditional Chinese Medicine; Qingdao, China). Written informed consent was obtained from each participant whose synovial fluids as well as peripheral blood were collected for the investigation. A total of 80 cases of patients with knee OA were mainly diagnosed using X-ray in combination with magnetic resonance image (MRI), and were recruited from those who were hospitalized from May 2014 to May 2017 in the Department of Rheumatology in the Affiliated Qingdao Hiser Hospital of Qingdao University. As corresponding healthy control, 80 cases of healthy patients whose peripheral blood only was obtained from the Department of Spine Surgery in Qingdao Hiser Hospital. The synovial fluid from healthy control was unavailable. It should be noted that, in order to be comparable for the detection results, the demographic characteristics including age, sex and education level were strictly matched. In addition, the patients who complained of pain in the knee had 


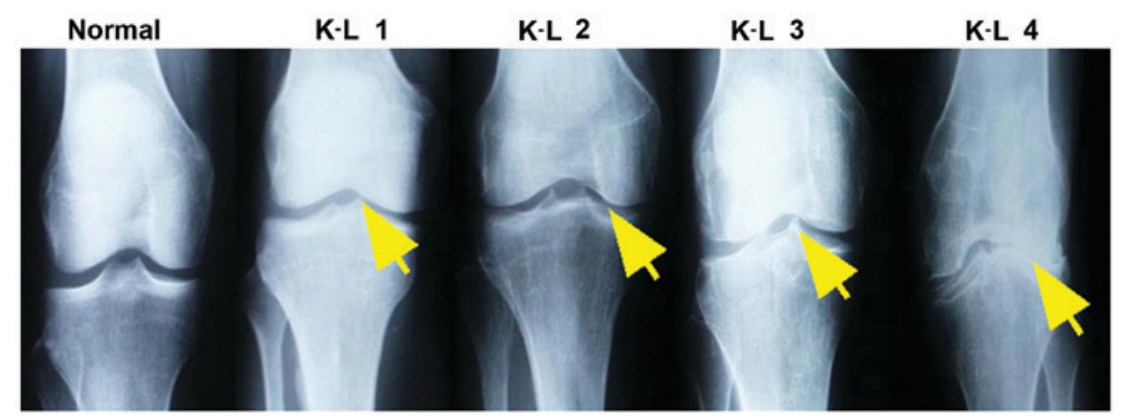

Figure 1. Roentgenograph of knee OA with different degree of severity ranging from K-L grade 1 to 4 diagnosed using X-ray. K-L 1, doubtful narrowing of joint space and possible bone spurs. K-L 2, presence of bone spurs and possible joint space narrowing on anteroposterior weight-bearing radiograph. K-L 3, multiple bone spurs are present, definite joint space narrowing, sclerosis and possible bony deformity. K-L 4, large spurs are present, marked joint space narrowing, severe sclerosis and definitely bony deformity. The bone spurs are present and indicated by the yellow arrow. OA, osteoarthritis; K-L, Kellgren-Lawrence.

Table I. Baseline characteristics of the enrolled 80 patients with knee OA.

\begin{tabular}{lc}
\hline Characteristics & Data \\
\hline Age (mean \pm SEM, years) & $58.0 \pm 8.4$ \\
Sex (\%) & \\
Men & $53(66.3)$ \\
Women & $27(33.7)$ \\
K-L grade (\%) & \\
I & $7(8.75)$ \\
II & $28(35)$ \\
III & $40(50)$ \\
IV & $5(6.25)$ \\
Pain evaluation & \\
Nominated activity VAS score & $50 \pm 20.35$ \\
No pain & 5 \\
Mild pain & 30 \\
Moderate pain & 35 \\
Severe pain & 10 \\
Knee injury gross assessment & \\
Synovitis & 12 \\
Cartilage lesion & 29 \\
Osteophytes appeared & 34 \\
Bone marrow lesion & 5 \\
\hline OA, osteoartritis; K-L, Kelggen-Lawrence; VAS, vis \\
\end{tabular}

OA, osteoarthritis; K-L, Kellgren-Lawrence; VAS, visual analogue scale. Range of VAS: No pain (0-4), mild (5-44), moderate (45-74) and severe pain (75-100).

Kellgren-Lawrence $(\mathrm{K}-\mathrm{L})$ grade $\geq 1$. In our enrollment, patients were excluded had they have any other form of arthritis; body mass index $(\mathrm{BMI})>24 \mathrm{~kg} / \mathrm{m}^{2}$; any disorder needing the use of systemic corticosteroids; history of knee replacement; chronic infectious disease and other chronic inflammation disease.

$K$-L and visual analogue scale (VAS) scoring. In consideration that the K-L grading scheme is the most widely used and accepted standard for measurement of knee OA here, scoring criteria using K-L was as follows (9): Grade 0, no radiographic features of OA are present; grade I, there is doubtful narrowing of joint space narrowing and possible osteophytes; grade II, there is presence of osteophytes and possible joint space narrowing on anteroposterior weight-bearing radiograph; grade III, multiple osteophytes were present, definite joint space narrowing, sclerosis and possible bony deformity; and grade IV, large osteophytes were present, marked joint space narrowing, severe sclerosis and definitely bony deformity (Fig. 1). The VAS scoring system used was based on reference (10), the range of VAS was from no pain (0-4 points), mild (5-44 points), moderate (45-74 points) to severe pain (75-100 points).

Reverse transcription-quantitative PCR (RT-qPCR). Extraction of total RNA from synovial fluids and peripheral whole blood was performed with TRIzol (Invitrogen; Thermo Fisher Scientific, Inc., Carlsbad, CA, USA) method following its manufacturer's protocol. To avoid possible degradation by RNase, total RNA was immediately reverse transcribed into cDNA using the iScript ${ }^{\mathrm{TM}}$ cDNA Synthesis kit according to the manufacturer's protocol. Reverse transcription-quantitative PCR (RT-qPCR) was performed using FastStart Universal SYBR-Green Master (ROX) Mix (2X) (Roche Diagnostics, Indianapolis, IN, USA), with $2.5 \mu \mathrm{l}$ cDNA per reaction in a total volume of $20 \mu \mathrm{l}$. RT-qPCR reactions were run in duplicates on the IQ5 ${ }^{\mathrm{TM}}$ real-time PCR system (Bio-Rad Laboratories, Hercules, CA, USA). The comparative Cq (cycle threshold) method $(-\Delta \Delta \mathrm{Cq})$ was applied for the quantification of gene expression (11). The values were normalized against $\beta$-actin as internal loading control. Standard curve as algorithm was used to calculate the relative expression of $\mathrm{CD}$ markers to $\beta$-actin. The results were expressed as fold-changes on mRNA level. The thermocycling conditions used were as follows: $30 \mathrm{sec}$ at $95^{\circ} \mathrm{C}$; at $60^{\circ} \mathrm{C}$; and at $72^{\circ} \mathrm{C}$ for 35 cycles. The following primers were used: CD86 forward, $5^{\prime}-\mathrm{CTG}$ CTCATCTATACACGGTTACC-3', and reverse, 5'-GGAAAC GTCGTACAGTTCTGTG-3'; CD163 forward, 5'-CAGGAA ACCAGTCCCAAACA-3', and reverse, 5'-AGCGACCTCCTC CATTTACC-3'; $\beta$-actin forward, 5'-CGTGACATTAAGGAG AAGCTG-3'; and reverse, 5'-CTAGAAGCATTTGCGGTG GAC-3'.

Flow cytometry. The expression of specific markers was investigated in monocytes from synovial fluid by fluorescenceactivated cell sorting analysis after surface or intracellular 
A

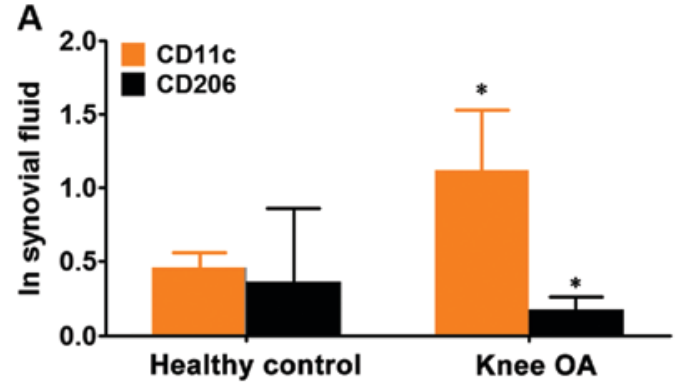

C

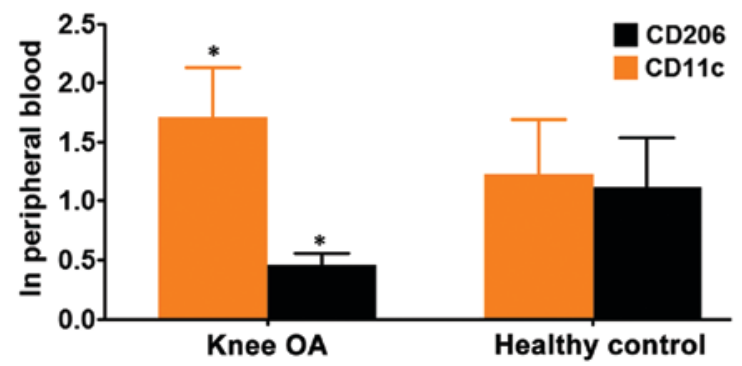

B

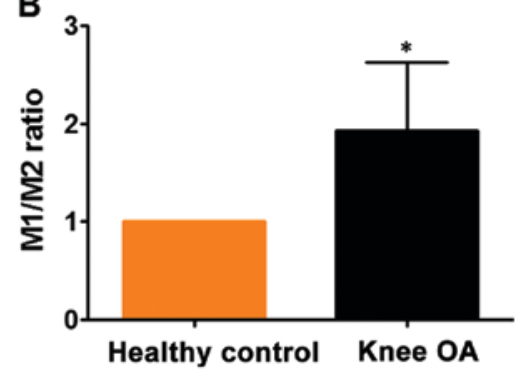

D

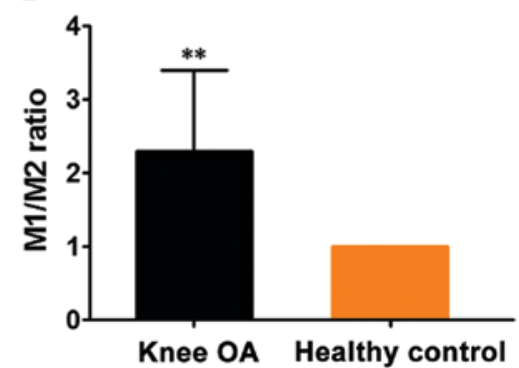

Figure 2. Detection of surface markers CD11c and CD206 expression was carried out using flow cytometry approach in the synovial fluids biopsied and peripheral blood from participants. (A) Expression of CD11c and CD206 surface markers of M1 and M2 macrophages was evaluated using flow cytometry in the synovial fluids biopsied from 35 patients with knee OA and paired healthy control. (B) Analysis of ratio of M1 to M2 macrophages in healthy controls and knee OA. (C) Likewise, expression of CD11c and CD206 surface markers was assayed using flow cytometry in the peripheral blood sampled from 80 cases of patients with knee OA and paired healthy control. (D) Similarly, ratio of M1 to M2 macrophages was calculated in healthy controls and knee OA. In light of the data being non-normal distribution, Mann-Whiney $\mathrm{U}$ test was used to analyze the statistical difference between healthy control and knee $\mathrm{OA}$. ${ }^{*} \mathrm{P}<0.05$, ${ }^{* *} \mathrm{P}<0.01$ in comparison with its control. OA, osteoarthritis.

staining with specific monobodies that were conjugated to different fluorescent dyes. For the extracellular staining, cells were washed in phosphate-buffered saline (PBS) containing $1 \%$ bovine serum albumin and $0.02 \%$ sodium azide and were incubated with specific antibodies for $30 \mathrm{~min}$ at $4^{\circ} \mathrm{C}$. Flow cytometry was performed using a FACS Canto (CytoFLEX; Beckman Coulter, CA, USA) and analyzed with Flowjo analysis software (Tree Star, Inc., Ashland, OR, USA). Specific monoclonal antibodies to human CD11c (Alexa Fluor $^{\circledR}$ 700; cat. no. 56-0116-42), CD206 (Alexa Fluor ${ }^{\circledR} 488$; cat. no. 53-2069-42), CD86 (Alexa Fluor ${ }^{\circledR} 488$; cat. no. A51007) and CD163 (PerCP-eFluor ${ }^{\circledR} 710$; cat. no. 46-1639-42) were all from Thermo Fisher Scientific, Inc. Staining with mouse IgG isotype control (PerCP-eFluor ${ }^{\circledR} 710$ labeled, cat. no. 46-4714; Thermo Fisher Scientific, Inc.) was used as control for analysis of monocytes in the monocyte gate.

Statistical analysis. The data are expressed as mean \pm standard error of means (SEM). The continuous data were subjected to Kolmogorov-Smirnov test for normal distribution. When the data of RT-qPCR detection were not normal distribution, Mann-Whitney U test was employed; the data from flow cytometry analysis had normal distribution, and independent sample t-test was used. Categorical data were analyzed using Chi-square or Fisher's exact test. SPSS 17.0 software version (SPSS Inc., Chicago, IL, USA) was used to analyze the statistical difference and GraphPad Prism 5.0 version (GraphPad Software, Inc., La Jolla, CA, USA) was employed to analyze and draw the relevant figures. $\mathrm{P}<0.05$ was considered to indicate a statistically significant difference.

\section{Results}

Patient characteristics. Although the fine needle aspiration biopsy of synovial fluids is a routine for patients with knee OA before therapy in our department, there were still some who were unwilling to have their synovial fluids biopsied and analyzed. Consequently, we retrieved the synovial fluids stored in liquid nitrogen device in the Biobank of The Affiliated Qingdao Hiser Hospital of Qingdao University in accordance with our requirement. Of the 80 patients with knee OA we enrolled from outset of the study, only 35 cases whose synovial fluids were available, yielding the median synovial fluid volume of $4 \mathrm{ml}$ (interquartitle range 3-8 ml). By contrast, peripheral blood was obtained from each participant, including healthy control. There was no significant difference in terms of age, knee injury, and VAS score between patients who did and those who did not have their synovial fluids biopsied and analyzed (Table I). In addition, Chi-square or Fisher's exact test samples were used to determine which exclusion of patients $\left(<500\right.$ cells $\left./ \mathrm{mm}^{3}\right)$. Eventually, eligible samples were obtained from 28 subjects. As shown in Table I, the mean \pm SD age of those 28 subjects was $58.0 \pm 8.4$ years, and $53 \%$ were men. The majority of participants had a K-L grade of 2 or 3 . The mean \pm SD VAS score at baseline was $50 \pm 20.35$.

The ratio of M1 to M2 macrophages is elevated in knee OA. Having made clear the baseline characteristics of patients with knee OA, subsequently, we determined the state of M1 and M2 macrophages in the synovial fluids and peripheral blood from participants, by performing flow cytometry analysis of given number of monocytes $(10,000$ cells) in these 28 cases of 

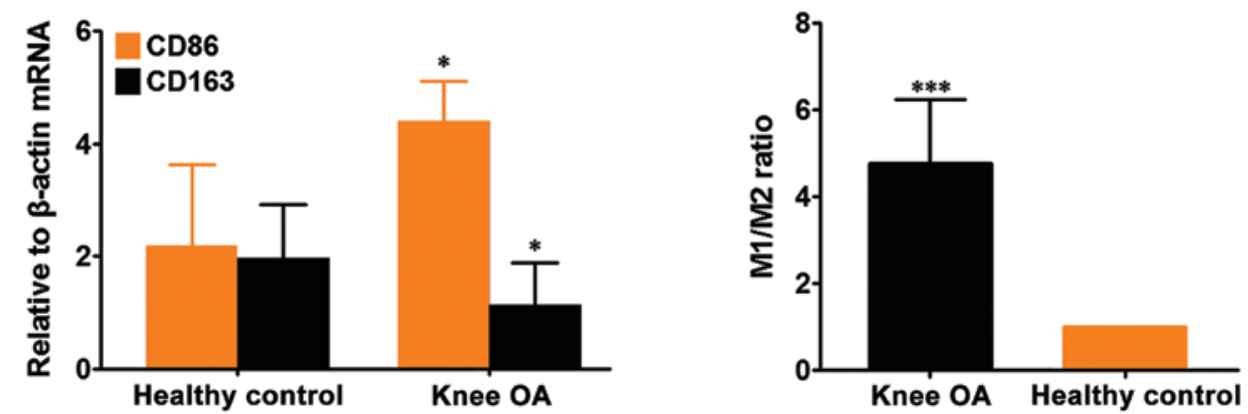

Figure 3. Detection of surface markers CD86 (typical surface marker of M1 macrophages) and CD163 (typical surface marker of M2 macrophages) expression was performed using RT-qPCR in the peripheral blood from 80 cases of patients with knee OA and its controls. Independent sample t-test was employed to analyze the difference of CD86 and CD163 expression between knee OA and healthy controls. ${ }^{*} \mathrm{P}<0.05,{ }^{* * *} \mathrm{P}<0.001$ compared with its control. The experiment was independently performed three times with each time having triplicate samples. OA, osteoarthritis

Table II. Association between ratio of M1/M2 macrophages and clinical variables at baseline.

\begin{tabular}{|c|c|c|c|c|c|}
\hline \multirow[b]{2}{*}{ Characteristics } & \multirow{2}{*}{$\begin{array}{l}\text { No. of } \\
\text { cases }\end{array}$} & \multicolumn{2}{|c|}{ Ratio of M1/M2 } & \multirow{2}{*}{$\begin{array}{c}\chi^{2} \\
\text { (or F) }\end{array}$} & \multirow[b]{2}{*}{ P-value } \\
\hline & & $1<\mathrm{R} \leq 1.5$ & $>1.5$ & & \\
\hline Age (years) & $58.0 \pm 8.4$ & 38 & 42 & & \\
\hline \multicolumn{6}{|l|}{$\operatorname{Sex}(\%)$} \\
\hline Men & $53(66.3 \%)$ & 25 & 28 & 0.053 & 1.000 \\
\hline Women & $27(33.7 \%)$ & 12 & 15 & & \\
\hline \multicolumn{6}{|l|}{ K-L grade $(\%)$} \\
\hline I & $7(8.75 \%)$ & 4 & 3 & 9.702 & 0.014 \\
\hline II & $28(35 \%)$ & 9 & 29 & & \\
\hline III & $40(50 \%)$ & 2 & 28 & & \\
\hline IV & $5(6.25 \%)$ & 0 & 5 & & \\
\hline \multicolumn{6}{|l|}{ Pain evaluation } \\
\hline Nominated activity & $50 \pm 20.35$ & & & & \\
\hline \multicolumn{6}{|l|}{ VAS score } \\
\hline No pain & 5 & 2 & 3 & 1.013 & 0.864 \\
\hline Mild pain & 30 & 16 & 14 & & \\
\hline Moderate pain & 35 & 21 & 14 & & \\
\hline Severe pain & 10 & 6 & 4 & & \\
\hline \multicolumn{6}{|l|}{$\begin{array}{l}\text { Knee injury } \\
\text { gross assessment }\end{array}$} \\
\hline Synovitis & 17 & 6 & 11 & 4.015 & 0.125 \\
\hline Cartilage lesion & 29 & 7 & 22 & & \\
\hline Osteophyte appear & 34 & 4 & 30 & & \\
\hline Bone marrow lesion & 0 & 0 & 0 & & \\
\hline
\end{tabular}

K-L, Kellgren-Lawrence; F, F-value.

synovial fluids using specific monoclonal antibody to human CD11c, surface marker of M1 subtype macrophage and CD206, surface marker of M2 macrophage. Flow cytometry analyses for expression of CD11c as M1 and CD206 as M2 marker exhibited that both M1 and M2 macrophages were present in the synovial fluids from knee OA. However, the variation in the case of expression of each marker between patients was large (data not shown), thus we calculated the total of M1 and M2 macrophages in each group. It showed that the expression CD11c was remarkably higher than CD208, suggesting that M1 macrophages predominantly existed in synovial fluids of knee OA compared with M2 macrophages (Fig. 2A and B). To verify the finding from synovial fluids of knee OA, we extended the flow cytometry analysis from synovial fluid to peripheral blood. It exhibited that both monocytes with cell surface markers CD11c and CD208 were also present in whole blood from knee OA and healthy control. The expression of $\mathrm{CD} 11 \mathrm{c}$ was shown to be significantly higher in knee OA than that of control whereas expression of CD208 was markedly lower in knee OA than that of control (Fig. 2C). Taken as a whole, the ratio of M1/M2 macrophages was pronouncedly higher in peripheral blood of knee OA relative to healthy control, indicating that there was an association between knee OA and ratio of M1/M2 macrophages (Fig. 2D). While no significant skewing ratio of M1/M2 macrophages can be observed within the control group (data not shown). Likewise, in consideration that there were several well-established surface markers for M1 and M2 macrophages except of CD11c and CD208 we've used, to further confirm the findings, we've employed RT-qPCR technique to detect the expression of CD86 (another established surface marker of M1 macrophage) and CD163 (M2 macrophage) in peripheral blood from the same cohort as CD11c and CD208. It was found that the ratio of M1/M2 macrophage was remarkably higher in knee OA than that of control, which was totally congruent with observations made by flow cytometry (Fig. 3). Taken together, the results demonstrated that the ratio of M1/M2 macrophages were significantly higher in knee OA, suggesting a relationship between disequilibrium of ratio M1 to M2 macrophages versus knee OA development.

The ratio of M1/M2 macrophages is associated with severity level of knee OA. Having understood the status of M1 and M2 macrophages in knee OA, next, we sought to analyze the clinical significance of the ratio of M1/M2 macrophages. Given that all the patients with knee OA enrolled in our study whose damage was scored using K-L grading system from the outset, we've tried to explore whether there was association between ratio of M1/M2 macrophages and progression of knee OA. In light of the large variation of expression of each marker between patients, the patients were stratified further into two groups in accordance with the value of the ratio of M1/M2 macrophages obtained by RT-qPCR in peripheral blood. The group whose ratio was $>1.5$ and group whose ratio was $\leq 1.5$ but $>1$. As shown in Table II, it can be seen that there was 
significant positive association between K-L scores and value of the ratio M1/M2 macrophages, suggesting that the greater the value of ratio the more severe the knee OA seems to be. Interestingly, no significant association was observed between VAS scores and the ratio of M1/M2 macrophages. No significant correlation was observed either between the ratio of M1/ M2 macrophages and knee injury gross assessment, although there seems to be a trend toward knee injury degree. These results suggest that the ratio $\mathrm{M} 1 / \mathrm{M} 2$ macrophages might be predictive of the severity of knee OA.

\section{Discussion}

This is the first report of the ratio of M1/M2 macrophages in synovial fluids of knee OA and clinical significance of the ratio of M1/M2 macrophages in knee OA. Ratio of M1/M2 macrophages was shown to be remarkably higher in knee OA compared with healthy control. We further showed that ratio of M1/M2 macrophages was significantly associated with K-L grading of knee OA, indicating that ratio of M1/M2 macrophages might be used as a predictor for the damage or severity for the knee with OA. These observations also suggest that ratio of M1/M2 macrophages in knee OA might be potentially used as a novel quantitative scoring system measuring the severity of knee OA. The comparison study therefore may be warranted.

OA was previously viewed as regular wear and tear of the body and the immune system was unlikely to be affected, but it is now generally appreciated to be a low-grade inflammatory disease affecting the whole joint. Despite extensive investigations in vitro mechanistic and in vivo animal models implied that macrophages were implicated (12) even found to play a certain role in the pathogenesis of knee OA $(12,13)$, there has been a paucity of direct evidence regarding the macrophages in the setting of knee OA except one recent study by Kraus et al (14) who provided direct evidence of macrophages in the synovial fluid of knee OA. Our present investigation was also prompted by the study in that although Kraus et al (14) quantitatively presented direct evidence for the involvement of macrophages in patients with symptomatic OA, nevertheless, they did not evaluate the status of M1 and M2 macrophages in patients with symptomatic OA in consideration that macrophages used to being subtyped into M1 and M2 types. Furthermore, they determined the localization of macrophages in joints, including finger joints, thumb bases, shoulders, toes and ankles, which is to say, the focus was not merely restricted to the knee. In this sense, our study totally differs from the previous one. From the outset of our study, we paid attention on OA limited to the knee, regardless of other types of OA. Besides, considering the two common subtypes of macrophage implicated in inflammation, we determined the status of both M1 and M2 macrophages in the knee OA, the former was believed to be pro-inflammatory while the later anti-inflammatory.

M1 macrophage was initially mentioned in the murine model of knee OA in the setting of obesity induced by high-fat diet $(6,13)$. The study by Wu et al (13) showed that M1 macrophages played a vital role in modulating the homeostasis of immune cells in the inflammation associated with the development of OA in obesity, which was suggestive of the important involvement of M1 macrophages in OA associated with obesity. However, no direct evidence has emerged regarding M1 macrophages in OA irrespective of the obesity factor. We tried to explore the status of M1 and M2 macrophages both in synovial fluids and whole blood from patients with OA, tentatively regardless of the confounder factor, obesity in $\mathrm{OA}$. We showed that the number of monocytes stained with specific surface marker pertaining to M1 macrophage was significant higher than monocytes with specific surface marker of M2 macrophage in knee OA, suggesting that M1 macrophages predominantly existed in knee OA compared with M2 macrophages. To avoid the confounder of obesity, the BMI index was set within $24 \mathrm{~kg} / \mathrm{m}^{2}$. Any case whose BMI was above the criteria was excluded. Under such condition, we presented that the ratio of M1/M2 macrophages was markedly higher in knee OA compared with healthy control. However, as for the role M1 and M2 macrophages mediated in knee OA, it remains to be further investigated.

The pathogenesis of knee OA is believed to be dependent on the impairment of chondrogenic differentiation. Previous report has identified that M1 macrophage polarization in the synovial fluid can exert a significant inhibition on the differentiation of chondrocytes (7), indicating that M1 macrophage plays the major role in the mediation of chondrogenesis. In our present investigation, the ratio of M1/M2 macrophages was shown to be pronouncedly associated with K-L grading of knee OA, which was fundamentally in line with the study by Kraus et al (14) reporting that knee OA severity was associated with activated macrophage in synovial fluid of systemic OA. Notably, despite the ratio was found to be unassociated with the knee injury gross assessment in our analysis, there seems to be a trend of increasing ratio towards the impairment degree of knee, which was indicative of the more the ratio the greater the severity degree tends to be. Nonetheless, caution is needed when interpreting our data because of the relatively small number of studied patients. To confirm our observations, we interchangeably used flow cytometry and RT-qPCR approach, which was complementary to each other in the detection of synovial and peripheral blood from knee OA and healthy controls.

There were still some limitations that should have been acknowledged from the outset of our study. First, the synovial fluid from knee OA to be analyzed was cryopreserved in liquid nitrogen for nearly 1 year. In view of relevant studies showing that storage in liquid nitrogen could have a bearing on the expression of surface markers of cells $(15,16)$, our results regarding flow cytometry detection of CD11c and CD206 in synovial fluids could be compromised. Secondly, our observations were made on the premise that all the participants whose BMI index was within $24 \mathrm{~kg} / \mathrm{m}^{2}$, that is obesity as a confounder was excluded at the outset. Consequently, extrapolation of direct ratio of M1/M2 macrophages in knee OA from this study should be approached with caution. Thirdly, more mechanistic data should have been given regarding M1 and M2 macrophages in the pathogenesis of knee OA, in addition to merely presenting of phenotype of macrophages.

In conclusion, to our knowledge this was the first report of the ratio of M1/M2 macrophages in knee OA. The imbalance of M1/M2 macrophages can feature the severity level of knee OA. 


\section{Acknowledgements}

Not applicable.

\section{Funding}

The present study was supported by Department of Rheumatology, The Affiliated Qingdao Hiser Hospital of Qingdao University (Qingdao, China).

\section{Availability of data and materials}

The datasets used and/or analyzed during the present study are available from the corresponding author on reasonable request.

\section{Authors' contributions}

BL drafted the manuscript and performed PCR. MZ and JZ was responsible for K-L and VAS scoring. MZ and HY contributed to flow cytometry. All authors read and approved the final study.

\section{Ethics approval and consent to participate}

The study was approved by the Ethics Committee of The Affiliated Qingdao Hiser Hospital of Qingdao University (Qingdao, China). Patients who participated in this research had complete clinical data. The signed informed consents were obtained from the patients or the guardians.

\section{Patient consent for publication}

Not applicable.

\section{Competing interests}

The authors declare that they have no competing interests.

\section{References}

1. Elias-Jones CJ, Farrow L, Reilly JH, Kerr S, Meek RM, Kelly MP, Campton JL and Millar NL: Inflammation and neovascularization in hip impingement: Not just wear and tear. Am J Sports Med 43: 1875-1881, 2015.

2. Scanzello CR: Role of low-grade inflammation in osteoarthritis. Curr Opin Rheumatol 29: 79-85, 2017.
3. Mobasheri A, Rayman MP, Gualillo O, Sellam J, van der Kraan P and Fearon U: The role of metabolism in the pathogenesis of osteoarthritis. Nat Rev Rheumatol 13: 302-311, 2017.

4. Makris EA, Gomoll AH, Malizos KN, Hu JC and Athanasiou KA: Repair and tissue engineering techniques for articular cartilage. Nat Rev Rheumatol 11: 21-34, 2015.

5. Gaffney L, Warren P, Wrona EA, Fisher MB and Freytes DO: Macrophages' role in tissue disease and regeneration. Results Probl Cell Differ 62: 245-271, 2017.

6. Barboza E, Hudson J, Chang WP, Kovats S, Towner RA, Silasi-Mansat R, Lupu F, Kent C and Griffin TM: Profibrotic infrapatellar fat pad remodeling without M1 macrophage polarization precedes knee osteoarthritis in mice with diet-induced obesity. Arthritis Rheumatol 69: 1221-1232, 2017.

7. Fahy N, de Vries-van Melle ML, Lehmann J, Wei W, Grotenhuis N, Farrell E, van der Kraan PM, Murphy JM, Bastiaansen-Jenniskens YM and van Osch GJ: Human osteoarthritic synovium impacts chondrogenic differentiation of mesenchymal stem cells via macrophage polarisation state. Osteoarthritis Cartilage 22: 1167-1175, 2014.

8. Ivanova EA and Orekhov AN: Monocyte activation in immunopathology: Cellular test for development of diagnostics and therapy. J Immunol Res 2016: 4789279, 2016.

9. Kellgren JH and Lawrence JS: Radiological assessment of osteo-arthrosis. Ann Rheum Dis 16: 494-502, 1957.

10. Gallasch $\mathrm{CH}$ and Alexandre NM: The measurement of musculoskeletal pain intensity: A comparison of four methods. Rev Gaucha Enferm 28: 260-265, 2007.

11. Livak KJ and Schmittgen TD: Analysis of relative gene expression data using real-time quantitative PCR and the 2(-Delta Delta C(T)) method. Methods 25: 402-408, 2001.

12. Daghestani HN, Pieper CF and Kraus VB: Soluble macrophage biomarkers indicate inflammatory phenotypes in patients with knee osteoarthritis. Arthritis Rheumatol 67: 956-965, 2015.

13. Wu CL, McNeill J, Goon K, Little D, Kimmerling K, Huebner J, Kraus V and Guilak F: Conditional macrophage depletion increases inflammation and does not inhibit the development of osteoarthritis in obese macrophage fas-induced apoptosis-transgenic mice. Arthritis Rheumatol 69: 1772-1783, 2017.

14. Kraus VB, McDaniel G, Huebner JL, Stabler TV, Pieper CF, Shipes SW, Petry NA, Low PS, Shen J, McNearney TA, et al: Direct in vivo evidence of activated macrophages in human osteoarthritis. Osteoarthritis Cartilage 24: 1613-1621, 2016.

15. Del Pino A, Ligero G, López MB, Navarro H, Carrillo JA, Pantoll SC and Díaz de la Guardia R: Morphology, cell viability, karyotype, expression of surface markers and plasticity of three human primary cell line cultures before and after the cryostorage in LN2 and GN2. Cryobiology 70: 1-8, 2015.

16. Davies OG, Smith AJ, Cooper PR, Shelton RM and Scheven BA: The effects of cryopreservation on cells isolated from adipose, bone marrow and dental pulp tissues. Cryobiology 69: 342-347, 2014.

This work is licensed under a Creative Common Attribution-NonCommercial-NoDerivatives 4.0 International (CC BY-NC-ND 4.0) License. 